\title{
ORBIT CLOSURE HIERARCHIES OF SKEW-SYMMETRIC MATRIX PENCILS*
}

\author{
ANDRII DMYTRYSHYN ${ }^{\dagger}$ AND BO KÅGSTRÖM ${ }^{\dagger}$
}

\begin{abstract}
We study how small perturbations of a skew-symmetric matrix pencil may change its canonical form under congruence. This problem is also known as the stratification problem of skew-symmetric matrix pencil orbits and bundles. In other words, we investigate when the closure of the congruence orbit (or bundle) of a skew-symmetric matrix pencil contains the congruence orbit (or bundle) of another skew-symmetric matrix pencil. The developed theory relies on our main theorem stating that a skew-symmetric matrix pencil $A-\lambda B$ can be approximated by pencils strictly equivalent to a skew-symmetric matrix pencil $C-\lambda D$ if and only if $A-\lambda B$ can be approximated by pencils congruent to $C-\lambda D$.
\end{abstract}

Key words. skew-symmetric matrix pencil, stratification, canonical structure information, orbit, bundle

AMS subject classifications. 15A21, 15A22, 65F15, 47A07

DOI. $10.1137 / 140956841$

1. Introduction. How canonical information changes under perturbations, e.g., the confluence and splitting of eigenvalues of a matrix pencil, are essential issues for understanding and predicting the behavior of the physical system described by the matrix pencil. In general, these problems are known to be ill-posed: small perturbations in the input data may lead to drastical changes in the answers. The ill-posedness stems from the fact that both the canonical forms and the associated reduction transformations are discontinuous functions of the entries of $A-\lambda B$. Therefore it is important to get knowledge about the canonical forms (or canonical structure information) of the pencils that are close to $A-\lambda B$. One way to investigate this problem is to construct the stratification (i.e., the closure hierarchy) of orbits and bundles of the pencils [17].

The stratification of matrix pencils under strict equivalence transformations [16, $17,18]$ as well as the stratification of controllability and observability pairs [19] are known. StratiGraph $[21,24]$ is a software tool for computing and visualization of such stratifications. The stratification of full normal rank matrix polynomials has been studied [22] and implemented in StratiGraph too (available as a prototype now).

Our objective is to stratify orbits and bundles of skew-symmetric matrix pencils, i.e., $A-\lambda B$ with $A^{T}=-A$ and $B^{T}=-B$, under congruence transformations. Skew-symmetric matrix pencils appear in several applications, e.g., the design of a passive velocity field controller [23], multisymplectic partial differential equations [3], and systems with bi-Hamiltonial structure [27]. Structure preserving linearizations of skew-symmetric matrix polynomials [25] allow us to investigate differential algebraic systems of higher orders via skew-symmetric matrix pencils. Canonical forms of skew-symmetric matrix pencils $[29,30]$ and the structured staircase algorithm $[2,4]$

${ }^{*}$ Received by the editors February 12, 2014; accepted for publication (in revised form) by F. M. Dopico September 11, 2014; published electronically November 18, 2014. This research was supported by the Swedish Research Council (VR) under grant A0581501 and by eSSENCE, a strategic collaborative e-Science programme funded by the Swedish government via VR. A preprint appears as report UMINF 14.02 .

http://www.siam.org/journals/simax/35-4/95684.html

${ }^{\dagger}$ Department of Computing Science and HPC2N, Umeå University, SE-901 87 Umeå, Sweden (andrii@cs.umu.se, bokg@cs.umu.se). 
have already been investigated. The codimensions of the congruence orbits of skewsymmetric matrix pencils are obtained from the solutions of the associated homogeneous systems of matrix equations in [14] (they can also be obtained by computing the numbers of independent parameters in the miniversal deformations [8]). The Matrix Canonical Structure toolbox for MATLAB was extended by the functions for calculating these codimensions [13].

In this paper, we develop the stratification theory for skew-symmetric matrix pencils, which (to our knowledge) is a novel contribution. For any problem dimension we construct the closure hierarchy graph for congruence orbits or bundles. Each node (vertex) of the graph represents an orbit (or a bundle) and each edge represents a cover/closure relation. In the graph, there is an upward path from a node representing $A-\lambda B$ to a node representing $C-\lambda D$ if and only if $A-\lambda B$ can be transformed by an arbitrarily small perturbation to a skew-symmetric matrix pencil whose canonical form is the one of $C-\lambda D$.

Some steps toward the understanding of stratifications of matrix pencils with mixed types of symmetries have been done recently, e.g., miniversal deformations [10, 11 ] and codimension computations [6, 7]; the stratifications of $2 \times 2$ and $3 \times 3$ matrices of bilinear forms which give the stratifications of $2 \times 2$ and $3 \times 3$ symmetric/skewsymmetric matrix pencils are given in [12]. For matrix pencils with two symmetric matrices see also $[9,15]$.

The rest of the paper is outlined as follows. In section 2, we review the Kronecker canonical form of a general matrix pencil $A-\lambda B$ under strict equivalence transformations, as well as the corresponding canonical form of skew-symmetric matrix pencils under structure preserving congruence transformations. We also state the conditions when a general matrix pencil can be skew-symmetrized. Section 3 is devoted to the derivation of the stratification of orbits of skew-symmetric matrix pencils. We obtain the new results by investigating and proving relations between using strict equivalence transformations versus congruence transformations. In section 4 , an algorithm based on the theory presented in section 3 for computing the orbit stratification of skew-symmetric matrix pencils is described. In addition, section 4.1 includes a stepby-step presentation and illustration of the derivation and computation of the closure hierarchy graph of the $4 \times 4$ case. Finally, the stratification of skew-symmetric matrix pencil bundles is discussed in section 5, where the $4 \times 4$ case is used again to illustrate similarities and differences between the orbit and bundle stratifications.

2. Preliminary results. We start by recalling the Kronecker canonical form (KCF) of general matrix pencils and canonical forms of skew-symmetric matrix pencils under congruence. All matrices that we consider have complex entries. Define $\overline{\mathbb{C}}:=$ $\mathbb{C} \cup \infty$.

For each $k=1,2, \ldots$, define the $k \times k$ matrices

$$
J_{k}(\mu):=\left[\begin{array}{cccc}
\mu & 1 & & \\
& \mu & \ddots & \\
& & \ddots & 1 \\
& & & \mu
\end{array}\right], \quad I_{k}:=\left[\begin{array}{cccc}
1 & & & \\
& 1 & & \\
& & \ddots & \\
& & & 1
\end{array}\right],
$$

and for each $k=0,1, \ldots$, define the $k \times(k+1)$ matrices

$$
F_{k}:=\left[\begin{array}{cccc}
0 & 1 & & \\
& \ddots & \ddots & \\
& & 0 & 1
\end{array}\right], \quad G_{k}:=\left[\begin{array}{cccc}
1 & 0 & & \\
& \ddots & \ddots & \\
& & 1 & 0
\end{array}\right] .
$$


All nonspecified entries of $J_{k}(\mu), I_{k}, F_{k}$, and $G_{k}$ are zeros.

An $m \times n$ matrix pencil $A-\lambda B$ is called strictly equivalent to $C-\lambda D$ if and only if there are nonsingular matrices $Q$ and $R$ such that $Q^{-1} A R=C$ and $Q^{-1} B R=D$. The set of matrix pencils strictly equivalent to $A-\lambda B$ forms a manifold in the complex $2 m n$ dimensional space. This manifold is the orbit of $A-\lambda B$ under the action of the group $G L_{m}(\mathbb{C}) \times G L_{n}(\mathbb{C})$ on the space of all matrix pencils by strict equivalence:

$$
\mathrm{O}_{A-\lambda B}^{e}=\left\{Q^{-1}(A-\lambda B) R: Q \in G L_{m}(\mathbb{C}), R \in G L_{n}(\mathbb{C})\right\} .
$$

The dimension of $\mathrm{O}_{A-\lambda B}^{e}$ is the dimension of the tangent space to this orbit

$$
\mathrm{T}_{A-\lambda B}^{e}:=\left\{(X A-A Y)-\lambda(X B-B Y): X \in \mathbb{C}^{m \times m}, Y \in \mathbb{C}^{n \times n}\right\}
$$

at the point $A-\lambda B$. The orthogonal complement to $\mathrm{T}_{A-\lambda B}^{e}$, with respect to the Frobenius inner product

$$
\langle A-\lambda B, C-\lambda D\rangle=\operatorname{trace}\left(A C^{*}+B D^{*}\right),
$$

is called the normal space to this orbit. The dimension of the normal space is the codimension of the strict equivalence orbit of $A-\lambda B$ and is equal to $2 m n$ minus the dimension of the strict equivalence orbit of $A-\lambda B$. Explicit expressions for the codimensions of strict equivalence orbits are presented in [5].

TheOrem 2.1 (see [20, sect. XII, 4]). Each $m \times n$ matrix pencil $A-\lambda B$ is strictly equivalent to a direct sum, uniquely determined up to permutation of summands, of pencils of the form

$$
\begin{aligned}
E_{k}(\mu) & :=J_{k}(\mu)-\lambda I_{k}, \text { in which } \mu \in \mathbb{C}, \quad E_{k}(\infty):=I_{k}-\lambda J_{k}(0), \\
L_{k} & :=F_{k}-\lambda G_{k}, \quad \text { and } \quad L_{k}^{T}:=F_{k}^{T}-\lambda G_{k}^{T} .
\end{aligned}
$$

The canonical form in Theorem 2.1 is known as the Kronecker canonical form. The blocks $E_{k}(\mu)$ and $E_{k}(\infty)$ correspond to the finite and infinite eigenvalues, respectively, and altogether form the regular part of $A-\lambda B$. The blocks $L_{k}$ and $L_{k}^{T}$ correspond to the column and row minimal indices, respectively, and form the singular part of the matrix pencil.

A sequence of integers $\mathcal{N}=\left(n_{1}, n_{2}, n_{3}, \ldots\right)$ such that $n_{1}+n_{2}+n_{3}+\cdots=n$ and $n_{1} \geqslant$ $n_{2} \geqslant \cdots \geqslant 0$ is called an integer partition of $n$ (for more details and references see [17]). For any $a \in \mathbb{Z}$ we define $\mathcal{N}+a$ as the integer partition $\left(n_{1}+a, n_{2}+a, n_{3}+a, \ldots\right)$ assuming that we take only $a$ such that $n_{i}+a$ for $i=1,2, \ldots$ are nonnegative and for positive $b \in \mathbb{Q}$ we define $b \mathcal{N}$ to be $\left(b n_{1}, b n_{2}, b n_{3}, \ldots\right)$ assuming that we take only $b$ such that $b n_{i}$ for $i=1,2, \ldots$ are integers. The difference of two integer partitions $\mathcal{N}=\left(n_{1}, n_{2}, n_{3}, \ldots\right)$ and $\mathcal{M}=\left(m_{1}, m_{2}, m_{3}, \ldots\right)$ is defined as $\mathcal{N}-\mathcal{M}=\left(n_{1}-m_{1}, n_{2}-m_{2}, n_{3}-m_{3}, \ldots\right)$; if in addition $n_{i}-m_{i} \geqslant n_{i+1}-m_{i+1} \geqslant 0$ for $i=1,2, \ldots$, then $\mathcal{N}-\mathcal{M}$ is an integer partition too. The set of all integer partitions forms a poset (even a lattice) with respect to the following order $\mathcal{N} \geqslant \mathcal{M}$ if and only if $n_{1}+n_{2}+\cdots+n_{i} \geqslant m_{1}+m_{2}+\cdots+m_{i}$ for $i \geqslant 1$.

With every matrix pencil $P=A-\lambda B$ (with eigenvalues $\mu_{j} \in \overline{\mathbb{C}}$ ) we associate the set of integer partitions $\mathcal{R}(P), \mathcal{L}(P)$, and $\left\{\mathcal{J}_{\mu_{j}}(P): j=1, \ldots, d\right\}$, where $d$ is the number of distinct eigenvalues of $P$ (e.g., see [17]). These partitions, known as the Weyr characteristics, are constructed as follows:

- For each distinct $\mu_{j}$ we have $\mathcal{J}_{\mu_{j}}(P)=\left(j_{1}^{\mu_{j}}(P), j_{2}^{\mu_{j}}(P), \ldots\right)$ : the $k$ th position is the number of Jordan blocks of the size greater than or equal to $k$, the position numeration starting from 1 . 
- $\mathcal{R}(P)=\left(r_{0}(P), r_{1}(P), \ldots\right)$ (or, respectively, $\left.\mathcal{L}(P)=\left(l_{0}(P), l_{1}(P), \ldots\right)\right)$ : the $k$ th position is the number of $L$-blocks (or, respectively, $L^{T}$-blocks) of the size greater than or equal to $k \times(k+1)$ (or, respectively, $(k+1) \times k$ ), i.e., with the associated minimal indices greater than or equal to $k$, the position numeration starting from 0 .

Example 2.2. Let $P=2 E_{3}\left(\mu_{1}\right) \oplus 2 E_{1}\left(\mu_{1}\right) \oplus 2 E_{2}(\infty) \oplus L_{4} \oplus L_{1} \oplus L_{4}^{T} \oplus L_{1}^{T}$ be a $24 \times 24$ matrix pencil in KCF. The associated partitions are

$$
\begin{aligned}
\mathcal{J}_{\mu_{1}}(P) & =(4,2,2), & \mathcal{J}_{\infty}(P) & =(2,2), \\
\mathcal{R}(P) & =(2,2,1,1,1), & \mathcal{L}(P) & =(2,2,1,1,1) .
\end{aligned}
$$

Matrices with specific characteristics should be treated with structure preserving transformations to keep their physical meaning. Therefore it is natural to consider skew-symmetric matrix pencils under congruence. An $n \times n$ skew-symmetric matrix pencil $A-\lambda B$ is called congruent to $C-\lambda D$ if and only if there is a nonsingular matrix $S$ such that $S^{T} A S=C$ and $S^{T} B S=D$. The set of matrix pencils congruent to a skew-symmetric matrix pencil $A-\lambda B$ forms a manifold in the complex $n^{2}-n$ dimensional space $(A$ has $n(n-1) / 2$ independent parameters and so does $B)$. This manifold is the orbit of $A-\lambda B$ under the action of the group $G L_{n}(\mathbb{C})$ on the space of skew-symmetric matrix pencils by congruence:

$$
\mathrm{O}_{A-\lambda B}^{c}=\left\{S^{T}(A-\lambda B) S: S \in G L_{n}(\mathbb{C})\right\} .
$$

The dimension of $\mathrm{O}_{A-\lambda B}^{c}$ is the dimension of the tangent space to this orbit

$$
\mathrm{T}_{A-\lambda B}^{c}:=\left\{\left(X^{T} A+A X\right)-\lambda\left(X^{T} B+B X\right): X \in \mathbb{C}^{n \times n}\right\}
$$

at the point $A-\lambda B$. The orthogonal complement (in the space of all skew-symmetric matrix pencils) to $\mathrm{T}_{A-\lambda B}^{c}$ with respect to (2.2) is the normal space to this orbit. The dimension of the normal space is the codimension of the congruence orbit of $A-\lambda B$ and is equal to $n^{2}-n$ minus the dimension of the congruence orbit of $A-\lambda B$.

Recently, explicit expressions for the codimensions of congruence orbits of skewsymmetric matrix pencils were derived in [14]. Note that these codimensions are different from the codimensions of the strict equivalence orbits of skew-symmetric matrix pencils (illustrated by the example in section 4.1).

TheOREM 2.3 (see [30]). Each skew-symmetric $n \times n$ matrix pencil $A-\lambda B$ is congruent to a direct sum, determined uniquely up to permutation of summands, of pencils of the form

$$
\begin{aligned}
H_{h}(\mu) & :=\left[\begin{array}{cc}
0 & J_{h}(\mu) \\
-J_{h}(\mu)^{T} & 0
\end{array}\right]-\lambda\left[\begin{array}{cc}
0 & I_{h} \\
-I_{h} & 0
\end{array}\right], \quad \mu \in \mathbb{C}, \\
K_{k} & :=\left[\begin{array}{cc}
0 & I_{k} \\
-I_{k} & 0
\end{array}\right]-\lambda\left[\begin{array}{cc}
0 & J_{k}(0) \\
-J_{k}(0)^{T} & 0
\end{array}\right], \\
M_{m} & :=\left[\begin{array}{cc}
0 & F_{m} \\
-F_{m}^{T} & 0
\end{array}\right]-\lambda\left[\begin{array}{cc}
0 & G_{m} \\
-G_{m}^{T} & 0
\end{array}\right] .
\end{aligned}
$$

Therefore every skew-symmetric pencil $A-\lambda B$ is congruent to one in the following direct sum form:

$$
A-\lambda B=\bigoplus_{j} \bigoplus_{i} H_{h_{i}}\left(\mu_{j}\right) \oplus \bigoplus_{i} K_{k_{i}} \oplus \bigoplus_{i} M_{m_{i}}
$$


where the first direct (double) sum corresponds to all $d$ distinct eigenvalues $\mu_{j}$.

We say that a matrix pencil can be skew-symmetrized if its strict equivalence orbit contains a skew-symmetric matrix pencil (e.g., $P$ from Example 2.2 can be skew-symmetrized).

THEOREM 2.4. A matrix pencil $P$ can be skew-symmetrized if and only if the following conditions hold:

1. For each distinct $\mu_{j}$ and every $k$ its KCF contains an even number of blocks $E_{k}\left(\mu_{j}\right)$.

2. For every $k$ its KCF contains an even number of blocks $E_{k}(\infty)$.

3. For every $k$ the number of blocks $L_{k}$ is equal to the number of blocks $L_{k}^{T}$ in its $K C F$.

Proof. The proof follows from the form of the canonical blocks of matrix pencils under congruence given in Theorem 2.3.

3. Orbit closure relations for skew-symmetric matrix pencils: Strict equivalence versus congruence. A classical result (see [20, Theorem 6, p. 41] or [26, Theorem 3, p. 275]) is that two skew-symmetric matrix pencils are strictly equivalent if and only if they are congruent. In this section, we generalize this fact, proving that a skew-symmetric matrix pencil $A-\lambda B$ can be approximated by pencils strictly equivalent to a skew-symmetric matrix pencil $C-\lambda D$ if and only if $A-\lambda B$ can be approximated by pencils congruent to $C-\lambda D$. First, we present three equivalent formulations of our main result: Theorems 3.1, 3.2, and 3.3. We also recall some known and provide some auxiliary results needed for the proof of the theorems. The proof of the main result is presented at the end of this section.

By $\overline{\mathcal{X}}$ we denote the closure of a set $\mathcal{X}$ in the Euclidean topology.

TheOREM 3.1. Let $A-\lambda B$ and $C-\lambda D$ be two skew-symmetric matrix pencils. Then the following holds:

$$
\overline{\mathrm{O}_{C-\lambda D}^{e}} \supset \mathrm{O}_{A-\lambda B}^{e} \text { if and only if } \overline{\mathrm{O}_{C-\lambda D}^{c}} \supset \mathrm{O}_{A-\lambda B}^{c} \text {. }
$$

Assuming $\mathrm{O}_{C-\lambda D}^{e} \neq \mathrm{O}_{A-\lambda B}^{e}$, the condition $\overline{\mathrm{O}_{C-\lambda D}^{e}} \supset \mathrm{O}_{A-\lambda B}^{e}$ implies that $\mathrm{O}_{A-\lambda B}^{e}$ is a part of the boundary of the orbit $\mathrm{O}_{C-\lambda D}^{e}$. Therefore there is an arbitrarily small perturbation of $A-\lambda B$ that brings it to a nearby pencil which is equivalent to $C-\lambda D$. The same is true for the congruence orbits. This leads to the following reformulation of Theorem 3.1.

TheOrem 3.2. Let $A-\lambda B$ and $C-\lambda D$ be two skew-symmetric matrix pencils. There exists an arbitrarily small (entrywise) matrix pencil $F-\lambda F^{\prime}$ and nonsingular matrices $Q$ and $R$ such that

$$
Q^{-1}\left(A+F-\lambda\left(B+F^{\prime}\right)\right) R=C-\lambda D
$$

if and only if there exists an arbitrarily small (entrywise) matrix pencil $\tilde{F}-\lambda \tilde{F}^{\prime}$, where $\tilde{F}^{T}=-\tilde{F}$ and $\tilde{F}^{\prime T}=-\tilde{F}^{\prime}$, and a nonsingular $S$ such that

$$
S^{T}\left(A+\tilde{F}-\lambda\left(B+\tilde{F}^{\prime}\right)\right) S=C-\lambda D .
$$

By the existence of arbitrarily small (entrywise) matrix pencils $F-\lambda F^{\prime}:=\left[f_{i j}-\right.$ $\left.\lambda f_{i j}^{\prime}\right]$ and $\tilde{F}-\lambda \tilde{F}^{\prime}:=\left[\tilde{f}_{i j}-\lambda \tilde{f}_{i j}^{\prime}\right]$ in Theorem 3.2 we mean that for every arbitrarily small $\varepsilon>0$ there exists matrix pencils with entries satisfying $\max _{i j}\left\{f_{i j}, f_{i j}^{\prime}, \tilde{f}_{i j}, \tilde{f}_{i j}^{\prime}\right\}<\varepsilon$.

From (3.2) we have $Q(C-\lambda D) R^{-1}-(A-\lambda B)=F-\lambda F^{\prime}$. The corresponding equality for congruence follows from (3.3). Since $F-\lambda F^{\prime}$ can be arbitrarily small we have another reformulation of Theorem 3.1. 
TheOrem 3.3. Let $A-\lambda B$ and $C-\lambda D$ be two skew-symmetric matrix pencils. There exists a sequence of nonsingular matrices $\left\{Q_{k}, R_{k}^{-1}\right\}$ such that

$$
Q_{k}(C-\lambda D) R_{k}^{-1} \rightarrow A-\lambda B
$$

if and only if there exists a sequence of nonsingular matrices $\left\{S_{k}\right\}$ such that

$$
S_{k}^{T}(C-\lambda D) S_{k} \rightarrow A-\lambda B .
$$

Remark 3.4. Note that if $\overline{\mathcal{X}} \supset \mathcal{Y}$ for some sets of matrices $\mathcal{X}$ and $\mathcal{Y}$, then $\overline{\mathcal{X}} \supset \overline{\mathcal{Y}}$. Thus $\overline{\mathrm{O}_{C-\lambda D}^{e}} \supset \mathrm{O}_{A-\lambda B}^{e}$ implies $\overline{\mathrm{O}_{C-\lambda D}^{e}} \supset \overline{\mathrm{O}_{A-\lambda B}^{e}}$. The same implication holds for the congruence orbits.

The rest of the section is dedicated to the proof of Theorem 3.1.

First we recall the result which describes all the possible changes in the KCF under small perturbations. These changes are structure transitions based on six different rules (see Theorem 3.5). By the structure transition $X \leadsto Y$ we mean that in the canonical form of a matrix pencil the blocks represented by $X$ are replaced by the blocks $Y$. Note that $X$ and $Y$ must have the same dimensions.

TheOrem 3.5 (see [1]). Let $P_{1}$ and $P_{2}$ be two matrix pencils such that $\overline{\mathrm{O}_{P_{1}}^{e}} \supset \mathrm{O}_{P_{2}}^{e}$ (i.e., there is an upward path from $P_{2}$ to $P_{1}$ in the corresponding closure hierarchy graph). Then $P_{1}$ can be obtained from $P_{2}$ changing canonical blocks of $P_{2}$ by applying a sequence of structure transitions, and each transition is of one of the six types below:

1. $L_{j-1} \oplus L_{k+1} \leadsto L_{j} \oplus L_{k}, 1 \leqslant j \leqslant k$;

2. $L_{j-1}^{T} \oplus L_{k+1}^{T} \leadsto L_{j}^{T} \oplus L_{k}^{T}, 1 \leqslant j \leqslant k$;

3. $L_{j} \oplus E_{k+1}(\mu) \leadsto L_{j+1} \oplus E_{k}(\mu), j, k=0,1,2, \ldots$ and $\mu \in \overline{\mathbb{C}}$;

4. $L_{j}^{T} \oplus E_{k+1}(\mu) \leadsto L_{j+1}^{T} \oplus E_{k}(\mu), j, k=0,1,2, \ldots$ and $\mu \in \overline{\mathbb{C}}$;

5. $E_{j}(\mu) \oplus E_{k}(\mu) \leadsto E_{j-1}(\mu) \oplus E_{k+1}(\mu), 1 \leqslant j \leqslant k$ and $\mu \in \overline{\mathbb{C}}$;

6. $L_{p} \oplus L_{q}^{T} \leadsto \oplus_{i=1}^{t} E_{k_{i}}\left(\mu_{i}\right)$ if $p+q+1=\sum_{i=1}^{t} k_{i}$ and $\mu_{i} \neq \mu_{i^{\prime}}$ for $i \neq i^{\prime}, \mu_{i} \in \overline{\mathbb{C}}$.

Remark 3.6. By Theorem 3.5 the number of column and row minimal indices, respectively, may decrease only when we go upward in the closure hierarchy.

Lemma 3.7. Let $P_{1}$ and $P_{2}$ be two skew-symmetric $n \times n$ matrix pencils and $\overline{\mathrm{O}_{P_{1}}^{e}} \supset \mathrm{O}_{P_{2}}^{e}$. Then the difference in the number of the column minimal indices (or associated $L$-blocks) of $P_{1}$ and $P_{2}$ is a nonnegative even number (might be zero), i.e., $r_{0}\left(P_{2}\right)-r_{0}\left(P_{1}\right)=2 s$ for some nonnegative integer $s$.

The same holds for the number of the row minimal indices (or associated $L^{T}$ blocks) of $P_{1}$ and $P_{2}$, i.e., $l_{0}\left(P_{2}\right)-l_{0}\left(P_{1}\right)=2 s$ too.

Proof. Let $P_{1}$ and $P_{2}$ have the canonical blocks $\left\{L_{p_{1}}, L_{p_{2}}, \ldots, L_{p_{r_{0}\left(P_{1}\right)}}\right\}$ and $\left\{L_{q_{1}}, L_{q_{2}}, \ldots, L_{q_{r_{0}\left(P_{2}\right)}}\right\}$, respectively, that correspond to the column minimal indices. By Theorem 2.3 the sets of the row minimal indices with associated canonical blocks are $\left\{L_{p_{1}}^{T}, L_{p_{2}}^{T}, \ldots, L_{p_{r_{0}\left(P_{1}\right)}^{T}}^{T}\right\}$ and $\left\{L_{q_{1}}^{T}, L_{q_{2}}^{T}, \ldots, L_{q_{r_{0}\left(P_{2}\right)}}^{T}\right\}$. Since the regular part of a skew-symmetric matrix pencil always has an even dimension (also see Theorem 2.3) we obtain

$$
\sum_{i=1}^{r_{0}\left(P_{1}\right)}\left(2 p_{i}+1\right) \equiv n(\bmod 2) \quad \text { and } \quad \sum_{i=1}^{r_{0}\left(P_{2}\right)}\left(2 q_{i}+1\right) \equiv n(\bmod 2),
$$

or equivalently

$$
r_{0}\left(P_{1}\right) \equiv n(\bmod 2) \quad \text { and } \quad r_{0}\left(P_{2}\right) \equiv n(\bmod 2),
$$


respectively. Subtracting the equations in (3.6) we get

$$
r_{0}\left(P_{2}\right)-r_{0}\left(P_{1}\right) \equiv 0(\bmod 2) .
$$

Since $\overline{\mathrm{O}_{P_{1}}^{e}} \supset \mathrm{O}_{P_{2}}^{e}$, we have that $r_{0}\left(P_{2}\right)>r_{0}\left(P_{1}\right)$ by Remark 3.6 (Theorem 3.5).

Obviously, the same holds for the row minimal indices $l_{0}\left(P_{1}\right)$ and $l_{0}\left(P_{2}\right)$.

Lemma 3.8. Let $P_{i}=\left[\begin{array}{cc}0 & W_{i} \\ -W_{i}^{T} & 0\end{array}\right]$, where $W_{i}=X_{i}-\lambda Y_{i}$ are arbitrary $p \times q$ pencils, $p+q=n$, for $i=1,2$. If $\overline{\mathrm{O}_{W_{1}}^{e}} \supset \mathrm{O}_{W_{2}}^{e}$, then $\overline{\mathrm{O}_{P_{1}}^{c}} \supset \mathrm{O}_{P_{2}}^{c}$.

Proof. Assuming $\overline{\mathrm{O}_{W_{1}}^{e}} \supset \mathrm{O}_{W_{2}}^{e}$, we have the existence of nonsingular $Q$ and $R$ and arbitrarily small (entrywise) $E$ such that

$$
Q^{-1}\left(W_{2}+E\right) R=W_{1} .
$$

After transposing both sides and multiplying with -1 , we get

$$
R^{T}\left(-W_{2}^{T}-E^{T}\right) Q^{-T}=-W_{1}^{T} .
$$

Altogether, we obtain

$$
\left[\begin{array}{cc}
Q^{-1} & 0 \\
0 & R^{T}
\end{array}\right]\left(\left[\begin{array}{cc}
0 & W_{2} \\
-W_{2}^{T} & 0
\end{array}\right]+\left[\begin{array}{cc}
0 & E \\
-E^{T} & 0
\end{array}\right]\right)\left[\begin{array}{cc}
Q^{-T} & 0 \\
0 & R
\end{array}\right]=\left[\begin{array}{cc}
0 & W_{1} \\
-W_{1}^{T} & 0
\end{array}\right],
$$

i.e., $\overline{\mathrm{O}_{P_{1}}^{c}} \supset \mathrm{O}_{P_{2}}^{c}$.

Define the normal rank [17] of an $m \times n$ matrix pencil $P$ as

$$
\operatorname{nrk}(P)=n-r_{0}(P)=m-l_{0}(P) .
$$

Recall that $r_{0}(P)$ and $l_{0}(P)$ are the total number of column and row minimal indices, respectively, in the KCF of $P$.

Lemma 3.9. Let $P$ be a matrix pencil, taken in the $K C F$, i.e., it is a direct sum of the blocks $E_{a_{i}}(\lambda), E_{b_{i}}(\infty), L_{c_{i}}$, and $L_{d_{i}}^{T}$; see Theorem 2.1. Then the normal rank of $P$ is equal to the sum of the indices, $a_{i}, b_{i}, c_{i}$, and $d_{i}$, of all its Kronecker canonical blocks.

The following theorem characterizes the closure relations in terms of the Kronecker invariants.

TheOREM 3.10 (see $[17,28]$ ). $\overline{\mathrm{O}_{P_{1}}^{e}} \supset \mathrm{O}_{P_{2}}^{e}$ if and only if the following relations hold:

$$
\begin{aligned}
\mathcal{R}\left(P_{1}\right)+\operatorname{nrk}\left(P_{1}\right) & \geqslant \mathcal{R}\left(P_{2}\right)+\operatorname{nrk}\left(P_{2}\right), \\
\mathcal{L}\left(P_{1}\right)+\operatorname{nrk}\left(P_{1}\right) & \geqslant \mathcal{L}\left(P_{2}\right)+\operatorname{nrk}\left(P_{2}\right), \\
\mathcal{J}_{\mu_{j}}\left(P_{1}\right)+r_{0}\left(P_{1}\right) & \leqslant \mathcal{J}_{\mu_{j}}\left(P_{2}\right)+r_{0}\left(P_{2}\right)
\end{aligned}
$$

for all $\mu_{j} \in \overline{\mathbb{C}}, j=1, \ldots, d$.

Equipped with the results in Theorems 2.4, 3.5, and 3.10, Lemmas 3.7, 3.8, and 3.9 , and the associated Remarks 3.4 and 3.6, we are ready to prove our main result.

Proof of Theorem 3.1. To show the sufficiency, note that (3.5), which is equivalent to the inclusion of the congruence orbits in (3.1), immediately implies (3.4), which is equivalent to the inclusion of the strict equivalence orbits in (3.1), with $Q_{k}:=S_{k}^{T}$ and $R_{k}^{-1}:=S_{k}$.

Let us prove the necessity. In the following, we use the compact notation $P_{1}:=$ $C-\lambda D$ and $P_{2}:=A-\lambda B$ for the two skew-symmetric pencils. By permutations of the 
rows and corresponding permutations of the columns, the matrix pencils $P_{i}, i=1,2$, taken in the canonical form (2.4) can be written as

$$
\tilde{P}_{i}=Q_{i}^{T} P_{i} Q_{i}=\left[\begin{array}{cc}
0 & W_{i} \\
-W_{i}^{T} & 0
\end{array}\right],
$$

where $W_{i}=X_{i}-\lambda Y_{i}$ is a $p \times q$ pencil, $p+q=n$, and $Q_{i}$ is a permutation matrix for $i=1,2$. Note that the choice of the pencils $W_{i}, i=1,2$ is not unique. Below we explain how to chose $W_{1}$ and $W_{2}$ in such a way that $\overline{\mathrm{O}_{W_{1}}^{e}} \supset \mathrm{O}_{W_{2}}^{e}$ and thus to get $\overline{\mathrm{O}_{\tilde{P} 1}^{c}} \tilde{O}^{\prime} \mathrm{O}_{\tilde{P}_{2}}^{c}$ by Lemma 3.8. Since $\tilde{P}_{1}$ and $\tilde{P}_{2}$ are congruent to $P_{1}$ and $P_{2}$, respectively (see (3.10)), we will have the desired inclusion $\overline{\mathrm{O}_{P_{1}}^{c}} \supset \mathrm{O}_{P_{2}}^{c}$.

We define the pencil $W_{1}$ to be a direct sum of the top-right corner blocks of the $H$-, $K$-, and $M$-summands (see Theorem 2.3) in the skew-symmetric canonical form (2.4) of $P_{1}$. In terms of the KCF presented in Theorem 2.1, these top-right corner blocks are the $E$-blocks for the $H$ - and $K$-summands and the $L$-blocks for the $M$ summands. All the remaining blocks, i.e., the bottom-left corner blocks of the $H$-, $K$-, and $M$-summands ( $-E^{T}$-blocks and $-L^{T}$-blocks in terms of KCF) in (2.4) of $P_{1}$, obviously form $-W_{1}^{T}$. The integer partitions associated with $W_{1}$ and their relations to the integer partitions associated with $P_{1}$ are as follows (the first elements of the partitions are used frequently and therefore listed in the right column):

$$
\begin{aligned}
\mathcal{R}\left(W_{1}\right) & =\mathcal{R}\left(P_{1}\right), & r_{0}\left(W_{1}\right) & =r_{0}\left(P_{1}\right), \\
\mathcal{L}\left(W_{1}\right) & =0, & l_{0}\left(W_{1}\right) & =0, \\
\mathcal{J}_{\mu_{j}}\left(W_{1}\right) & =\frac{1}{2} \mathcal{J}_{\mu_{j}}\left(P_{1}\right), j=1, \ldots, d, & j_{1}^{\mu_{j}}\left(W_{1}\right) & =\frac{1}{2} j_{1}^{\mu_{j}}\left(P_{1}\right), j=1, \ldots, d .
\end{aligned}
$$

By Lemma 3.7, the number of $L$-blocks of $P_{1}$ is smaller by an even nonnegative integer, denoted by $2 s$, compared to $P_{2}$, i.e.,

$$
r_{0}\left(P_{1}\right)+2 s=r_{0}\left(P_{2}\right) .
$$

Then from Theorem 2.4, it follows that the number of $L^{T}$-blocks of $P_{1}$ is also smaller by $2 s$ compared to $P_{2}$, i.e., $l_{0}\left(P_{1}\right)+2 s=l_{0}\left(P_{2}\right)$. In fact, $P_{1}$ has $-L^{T}$-blocks but since $-L_{k}^{T}$ is strictly equivalent to $L_{k}^{T}$ we can omit the minus signs.

Now we define the pencil $W_{2}$ to be a direct sum of all the top-right corner blocks of the $H$ - and $K$-summands in the skew-symmetric canonical form (2.4) of $P_{2}$, the bottom-left corner blocks of the $s$ largest $M$-summands (i.e., the $s$ largest $L^{T}$-blocks) in (2.4) of $P_{2}$, and the top-right corner blocks of the $r_{0}\left(P_{2}\right)-s$ smallest $M$-summands (i.e., the $r_{0}\left(P_{2}\right)-s$ smallest $L$-blocks) in (2.4) of $P_{2}$. All the remaining blocks form $-W_{2}^{T}$. The integer partitions associated with $W_{2}$ and their relations to the integer partitions associated with $P_{2}$ are as follows (with the first elements of the partitions in the right column):

$$
\begin{aligned}
\mathcal{R}\left(W_{2}\right) & =\mathcal{R}\left(P_{2}\right)-\mathcal{S}_{\mathcal{R}}, & r_{0}\left(W_{2}\right) & =r_{0}\left(P_{2}\right)-s, \\
\mathcal{L}\left(W_{2}\right) & =\mathcal{S}_{\mathcal{L}}, & l_{0}\left(W_{2}\right) & =s, \\
\mathcal{J}_{\mu_{j}}\left(W_{2}\right) & =\frac{1}{2} \mathcal{J}_{\mu_{j}}\left(P_{2}\right), j=1, \ldots, d, & j_{1}^{\mu_{j}}\left(W_{2}\right) & =\frac{1}{2} j_{1}^{\mu_{j}}\left(P_{2}\right), j=1, \ldots, d,
\end{aligned}
$$

where the $s$ largest $L^{T}$-blocks in $P_{2}$, moved to $W_{2}$, form $\mathcal{L}\left(W_{2}\right)=\mathcal{S}_{\mathcal{L}}$ and the $r_{0}\left(P_{2}\right)-s$ smallest $L$-blocks in $P_{2}$ moved to $W_{2}$ form $\mathcal{R}\left(W_{2}\right)=\mathcal{R}\left(P_{2}\right)-\mathcal{S}_{\mathcal{R}}$. Note that $\mathcal{S}_{\mathcal{R}}=\mathcal{S}_{\mathcal{L}}$ 
and we use both partitions to specify whether the partition corresponds to $L$ - or $L^{T}$ blocks. Let us also recall that the minus sign between partitions (i.e., elementwise subtraction) represents the following: from the pencil that corresponds to $\mathcal{R}\left(P_{2}\right)$ we take away all the canonical summands that are in the pencil corresponding to $\mathcal{S}_{\mathcal{R}}$. We can express the normal ranks of $P_{1}, P_{2}, W_{1}$, and $W_{2}$ via $n, s$, and $r_{0}\left(P_{2}\right)$. By definition,

$$
\operatorname{nrk}\left(P_{1}\right)=n-r_{0}\left(P_{2}\right)+2 s \text { and } \operatorname{nrk}\left(P_{2}\right)=n-r_{0}\left(P_{2}\right) .
$$

Recall that the sets of the indices of $L$ - and $L^{T}$-blocks are exactly the same $\left(\mathcal{R}\left(P_{2}\right)=\right.$ $\mathcal{L}\left(P_{2}\right)$ ); see Theorem 2.4. The indices (but not the blocks) are equally distributed in both cases, i.e., between the blocks $W_{1}$ and $-W_{1}^{T}$ in $P_{1}$, and between the blocks $W_{2}$ and $-W_{2}^{T}$ in $P_{2}$. Being more precise, a block $J_{k}(\mu)$ is in $W_{i}$ if and only if there is a block $-J_{k}^{T}(\mu)$ in $-W_{i}^{T}$ and a block $L_{k}$ (or $L_{k}^{T}$ ) is in $W_{i}$ if and only if a block $-L_{k}^{T}$ (or $\left.-L_{k}\right)$ is in $-W_{i}^{T}$. Therefore, by the construction of $W_{1}$ and $W_{2}$, and by Lemma 3.9 we have

$$
\operatorname{nrk}\left(W_{1}\right)=\frac{n-r_{0}\left(P_{2}\right)}{2}+s \quad \text { and } \operatorname{nrk}\left(W_{2}\right)=\frac{n-r_{0}\left(P_{2}\right)}{2} .
$$

Since $\overline{\mathrm{O}_{P_{1}}^{e}} \supset \mathrm{O}_{P_{2}}^{e}$, the conditions (3.7)-(3.9) hold by Theorem 3.10. By (3.7) we have

$$
\mathcal{R}\left(P_{1}\right)+n-r_{0}\left(P_{2}\right)+2 s \geqslant \mathcal{R}\left(P_{2}\right)+n-r_{0}\left(P_{2}\right) .
$$

Subtracting $n-r_{0}\left(P_{2}\right)$ from both sides we obtain

$$
\mathcal{R}\left(P_{1}\right)+2 s \geqslant \mathcal{R}\left(P_{2}\right) .
$$

The last majorization is equivalent to the following set of inequalities for the partition entries:

$$
\sum_{k=0}^{j}\left(r_{k}\left(P_{1}\right)+2 s\right) \geqslant \sum_{k=0}^{j} r_{k}\left(P_{2}\right), \quad j=0,1,2, \ldots, n .
$$

To prove the sought majorization (3.15), we rewrite the set (3.12) as follows:

$$
\sum_{k=0}^{j}\left(r_{k}\left(P_{1}\right)+s\right) \geqslant \sum_{k=0}^{j}\left(r_{k}\left(P_{2}\right)-s\right), \quad j=0,1,2, \ldots, n .
$$

First, the partition that corresponds to subtracting the $s$ largest blocks from $\mathcal{R}\left(P_{2}\right)$ is

$$
\mathcal{R}\left(P_{2}\right)-\mathcal{S}_{\mathcal{R}}=\left(r_{0}\left(P_{2}\right)-s, r_{1}\left(P_{2}\right)-s, \ldots, r_{\gamma}\left(P_{2}\right)-s, 0, \ldots, 0\right),
$$

where $\gamma$ is the position of the last nonzero entry in the partition, i.e., $r_{\gamma}\left(P_{2}\right)-s>0$ (recall that we start the position numeration from 0 ). Now, we obtain

$$
\mathcal{R}\left(P_{1}\right)+s \geqslant \mathcal{R}\left(P_{2}\right)-\mathcal{S}_{\mathcal{R}},
$$

since the corresponding inequalities for $j=0, \ldots, \gamma$ are the same as in (3.13) and for $j=\gamma+1, \ldots, n$ they follow immediately from (3.14); the corresponding $j$ th entries of the partition $\mathcal{R}\left(P_{2}\right)-\mathcal{S}_{\mathcal{R}}$ are zeros. In terms of partitions for $W_{i}, i=1,2$, we have

$$
\begin{aligned}
\mathcal{R}\left(W_{1}\right)+s & \geqslant \mathcal{R}\left(W_{2}\right), \\
\mathcal{R}\left(W_{1}\right)+\frac{n-r_{0}\left(P_{2}\right)}{2}+s & \geqslant \mathcal{R}\left(W_{2}\right)+\frac{n-r_{0}\left(P_{2}\right)}{2},
\end{aligned}
$$


or equivalently

$$
\mathcal{R}\left(W_{1}\right)+\operatorname{nrk}\left(W_{1}\right) \geqslant \mathcal{R}\left(W_{2}\right)+\operatorname{nrk}\left(W_{2}\right) .
$$

To prove the majorization for the $\mathcal{L}$-partitions we note that $(s, s, \ldots) \geqslant \mathcal{S}_{\mathcal{L}}$. Therefore

$$
\begin{aligned}
\mathcal{L}\left(W_{1}\right)+s & \geqslant \mathcal{L}\left(W_{2}\right), \\
\mathcal{L}\left(W_{1}\right)+\frac{n-r_{0}\left(P_{2}\right)}{2}+s & \geqslant \mathcal{L}\left(W_{2}\right)+\frac{n-r_{0}\left(P_{2}\right)}{2},
\end{aligned}
$$

or equivalently using the normal ranks,

$$
\mathcal{L}\left(W_{1}\right)+\operatorname{nrk}\left(W_{1}\right) \geqslant \mathcal{L}\left(W_{2}\right)+\operatorname{nrk}\left(W_{2}\right) .
$$

Using (3.9) for each distinct $\mu_{j}$ we have

$$
\begin{aligned}
\mathcal{J}_{\mu_{j}}\left(P_{1}\right)+r_{0}\left(P_{2}\right)-2 s & \leqslant \mathcal{J}_{\mu_{j}}\left(P_{2}\right)+r_{0}\left(P_{2}\right), \\
2 \mathcal{J}_{\mu_{j}}\left(W_{1}\right)-2 s & \leqslant 2 \mathcal{J}_{\mu_{j}}\left(W_{2}\right), \\
\mathcal{J}_{\mu_{j}}\left(W_{1}\right)+r_{0}\left(W_{2}\right)-s & \leqslant \mathcal{J}_{\mu_{j}}\left(W_{2}\right)+r_{0}\left(W_{2}\right) .
\end{aligned}
$$

By (3.11) we have that $r_{0}\left(W_{2}\right)-s=r_{0}\left(W_{1}\right)$. Therefore

$$
\mathcal{J}_{\mu_{j}}\left(W_{1}\right)+r_{0}\left(W_{1}\right) \leqslant \mathcal{J}_{\mu_{j}}\left(W_{2}\right)+r_{0}\left(W_{2}\right) .
$$

Summing up, (3.16), (3.17), and (3.18) imply $\overline{\mathrm{O}_{W_{1}}^{e}} \supset \mathrm{O}_{W_{2}}^{e}$ by Theorem 3.10.

4. Orbit stratification of skew-symmetric matrix pencils. The stratification algorithm of complex skew-symmetric matrix pencils under congruence is mainly based on Theorem 3.1 and the closure hierarchy graph for matrix pencils under strict equivalence.

Let us recall that in the orbit stratification graph each node represents an orbit and each edge represents the closure/cover relation. There is an upward path from $\mathrm{O}_{A-\lambda B}$ to $\mathrm{O}_{C-\lambda D}$ if and only if $\overline{\mathrm{O}_{C-\lambda D}} \supset \mathrm{O}_{A-\lambda B}$ (here all the orbits are either under strict equivalence or under congruence).

ALGORITHM 4.1 (stratification of skew-symmetric matrix pencils).

Step 1. Construct the stratification of $n \times n$ matrix pencils under strict equivalence $[16,17]$ (e.g., using StratiGraph [21]).

Step 2. Extract from the stratification in Step 1 the nodes corresponding to the matrix pencils that can be skew-symmetrized (Theorem 2.4). They are in one-to-one correspondence with the congruence orbits of skew-symmetric matrix pencils.

Step 3. Replace the KCFs with the canonical forms under congruence (it is possible because we chose only the orbits of matrix pencils that can be skew-symmetrized) and place them according to the codimensions computed separately [14].

Step 4. Put an edge in between two nodes obtained in Step 3 if there is an upward path (may be through other nodes) in between the corresponding orbits in the graph obtained in Step 1 and no edge otherwise. We do not put an edge in between two nodes (obtained at Step 3 ) if there is already an upward path from one to another via some other nodes.

In the following, we explain why the obtained subgraph is the stratification of skew-symmetric matrix pencils under congruence, i.e., the correctness of Algorithm 4.1. 
Step 2 is justified by the fact that two skew-symmetric matrix pencils are congruent if and only if they are equivalent [26]. Thus all congruent skew-symmetric matrix pencils have the same KCF which is easily determined from the canonical form under congruence (see Theorem 2.3). Matrix pencils that can be skew-symmetrized can be found using Theorem 2.4 .

The legality of Step 4 follows from Theorem 3.1, which ensures that for each pair of two skew-symmetric $n \times n$ matrix pencils $A-\lambda B$ and $C-\lambda D$ there is an upward path from $A-\lambda B$ to $C-\lambda D$ in the stratification of $n \times n$ matrix pencil orbits under strict equivalence if and only if there is an upward path from $A-\lambda B$ to $C-\lambda D$ in the stratification of skew-symmetric $n \times n$ matrix pencil orbits under congruence.

4.1. Orbit stratification of the $4 \times 4$ case. Using Algorithm 4.1 we stratify orbits of skew-symmetric $4 \times 4$ matrix pencils.

Step 1 . The stratification (or closure hierarchy graph) of $4 \times 4$ matrix pencils under strict equivalence is constructed using StratiGraph; see Figure 1. Nodes corresponding to orbits with the same codimensions (left column) are listed on the same level in the graph.

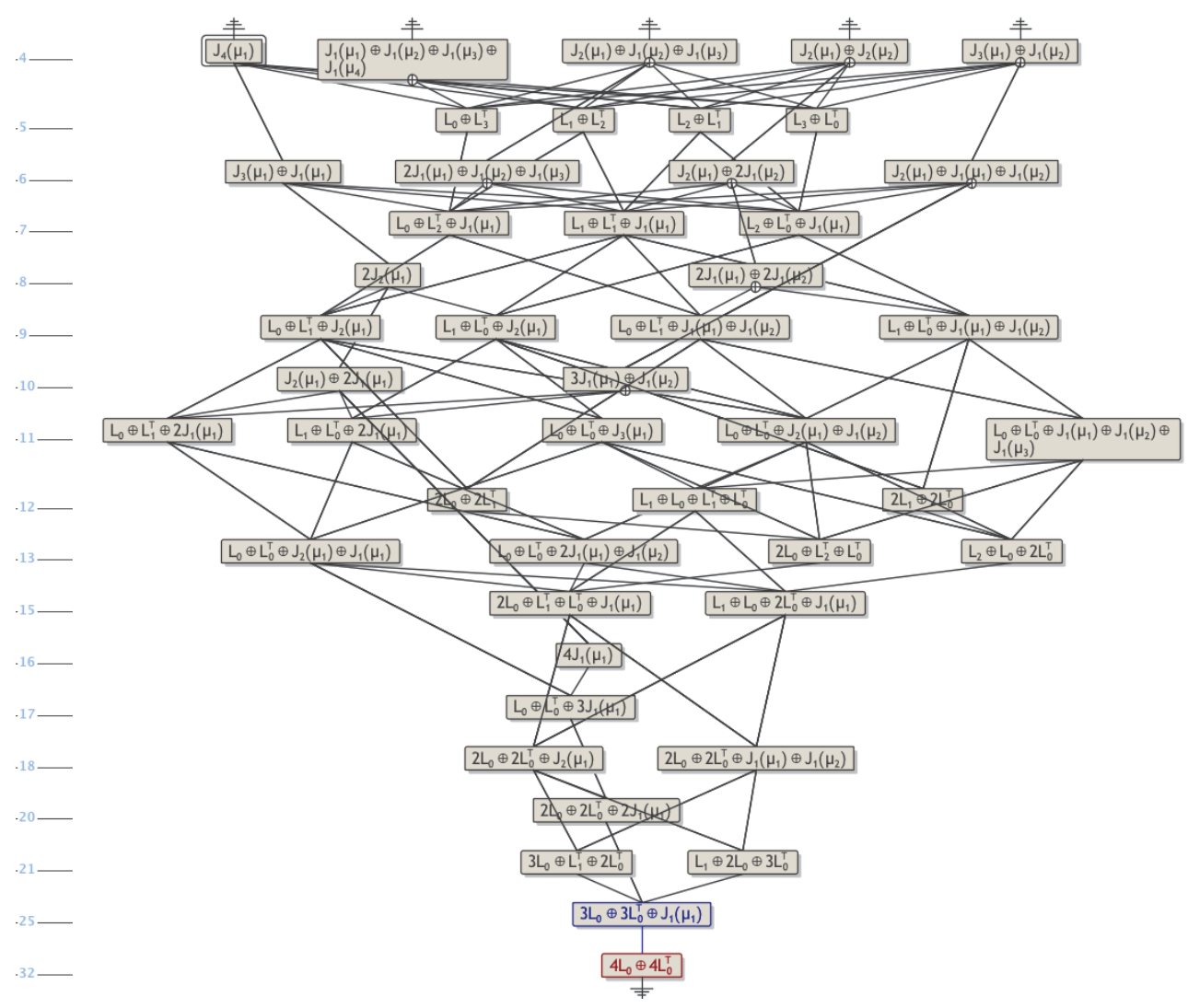

Fig. 1. Orbit stratification of $4 \times 4$ matrix pencils under strict equivalence. In the bottom of the graph there is the most degenerate orbit corresponding to the zero pencil. In the top of the graph there are five types of the most generic orbits. The other orbits are placed in between with respect to their codimensions (4-32 listed on the left). Note that in StratiGraph pencils $J_{n}(\mu)-\lambda I_{n}$ and $I_{n}-\lambda J_{n}(0)$ are denoted by $J_{n}(\mu)$ in which $\mu \in \overline{\mathbb{C}}, F_{n}-\lambda G_{n}$ by $L_{n}$, and $F_{n}^{T}-\lambda G_{n}^{T}$ by $L_{n}^{T}$. 
Step 2. The matrix pencils in Figure 1 (with codimensions) that can be skewsymmetrized are

$$
\begin{array}{llll}
4 L_{0} \oplus 4 L_{0}^{T}, & \text { codim. 32; } & L_{1} \oplus L_{0} \oplus L_{1}^{T} \oplus L_{0}^{T}, & \text { codim. 12; } \\
2 L_{0} \oplus 2 L_{0}^{T} \oplus 2 J_{1}\left(\mu_{1}\right), & \text { codim. 20; } & 2 J_{2}\left(\mu_{1}\right), & \text { codim. 8; } \\
4 J_{1}\left(\mu_{1}\right), & \text { codim. 16; } & 2 J_{1}\left(\mu_{1}\right) \oplus 2 J_{1}\left(\mu_{2}\right), & \text { codim. 8. }
\end{array}
$$

Step 3. We replace the KCFs by the canonical forms under congruence. For example, $2 L_{0} \oplus 2 L_{0}^{T} \oplus 2 J_{1}\left(\mu_{1}\right)$ is replaced by $2 M_{0} \oplus H_{1}\left(\mu_{1}\right)$ and $L_{1} \oplus L_{0} \oplus L_{1}^{T} \oplus L_{0}^{T}$ is replaced by $M_{1} \oplus M_{0}$. We also compute the corresponding codimensions under congruence using formulas from [14].

Step 4 . We check all the possible pairs of nodes. For example, there is a path from $2 L_{0} \oplus 2 L_{0}^{T} \oplus 2 J_{1}\left(\mu_{1}\right)$ to $L_{1} \oplus L_{0} \oplus L_{1}^{T} \oplus L_{0}^{T}$ (it is going through the orbits $2 L_{0} \oplus 2 L_{0}^{T} \oplus J_{2}\left(\mu_{1}\right)$ and $\left.2 L_{0} \oplus L_{0}^{T} \oplus L_{1}^{T} \oplus J_{1}\left(\mu_{1}\right)\right)$; therefore we have an edge from $2 M_{0} \oplus H_{1}\left(\mu_{1}\right)$ to $M_{1} \oplus M_{0}$ in the stratification of skew-symmetric matrix pencils under congruence. We leave the straightforward verification of the other edges (or their absence) to the reader. In summary, we get the stratification with the congruence orbit codimensions listed to the right:

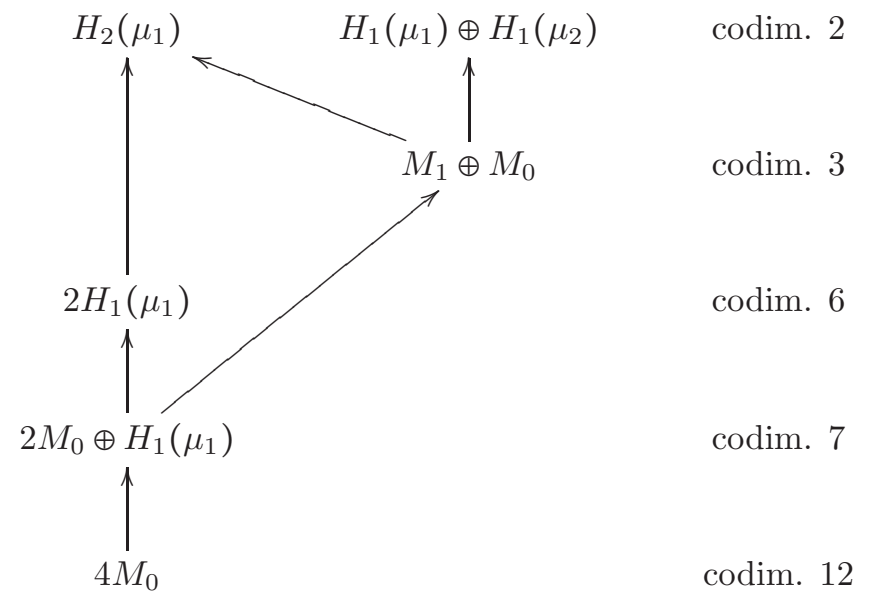

Since $\mu_{1}$ and $\mu_{2}$ represent distinct eigenvalues we can take either $\mu_{1}=\infty$ or $\mu_{2}=\infty$. In order to correspond to the notation in Theorem 2.3, and if we take one eigenvalue being infinite, we have to replace $H_{1}\left(\mu_{1}\right)$ and $H_{2}\left(\mu_{1}\right)$ by $K_{1}$ and $K_{2}$, respectively, or $H_{1}\left(\mu_{2}\right)$ by $K_{1}$ in the stratification graph above.

The complete stratification process is illustrated in Figure 2.

5. Bundle stratification of skew-symmetric matrix pencils. As in the case of matrix pencils under strict equivalence $[16,17]$, we also consider stratification of congruence bundles. A bundle $\mathrm{B}_{A-\lambda B}^{c}$ is a union of skew-symmetric matrix pencil orbits with the same singular structures and the same Jordan structures except that the distinct eigenvalues may be different. This definition of bundle is analogous to the one for matrix pencils under strict equivalence [17]. Therefore we have that two skew-symmetric pencils are in the same bundle under strict equivalence if and only if they are in the same bundle under congruence. This, together with Theorem 3.1, ensures that for skew-symmetric matrix pencils, the stratification algorithm for bundles is analogous to the one for orbits (Algorithm 4.1). So we extract the skewsymmetrized bundles from the stratification of matrix pencil bundles and put an 


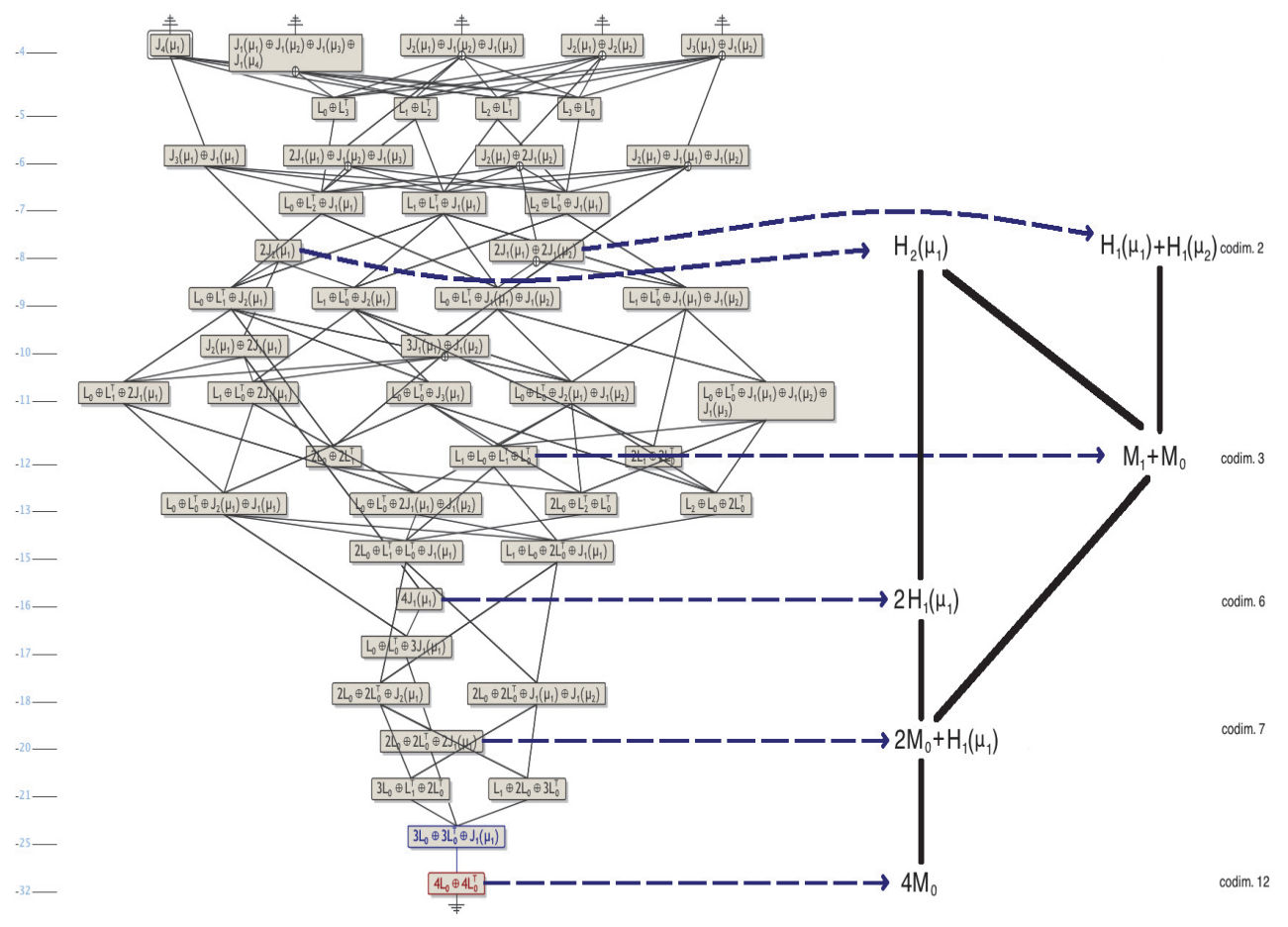

FIG. 2. Orbit stratification of skew-symmetric $4 \times 4$ matrix pencils under congruence (right graph) extracted from the orbit stratification of all $4 \times 4$ matrix pencils under strict equivalence (left graph) using Algorithm 4.1.

edge between two of them if there was a path between them in the matrix pencil graph. As in the graphs for orbits we do not write an edge between two nodes if there is already a path from one to another via some other nodes. In addition, the codimension of a skew-symmetric matrix pencil bundle of $A-\lambda B$ under congruence is defined as

$$
\operatorname{codim} \mathrm{B}_{A-\lambda B}^{c}=\operatorname{codim} \mathrm{O}_{A-\lambda B}^{c}-\#\{\text { distinct eigenvalues of } A-\lambda B\} .
$$

Example 5.1. In Figure 3, we stratify bundles of skew-symmetric $4 \times 4$ matrix pencils. Each node in the closure hierarchy graph to the right represents a bundle under congruence and each edge a closure/cover relation. Perturbing arbitrarily small an element from a given bundle in the closure hierarchy we can get an element of any bundle to which we have an upward path in the graph.

Notably, in the orbit stratification the eigenvalues may appear and disappear but they are fixed (cannot change). By contrast, in the bundle stratification the eigenvalues may coalesce or split apart. As a consequence, each of the two bundle graphs in Figure 3 has only one most generic node (bundles with four and two distinct eigenvalues, respectively), while the two orbit graphs in Figure 2 have more than one most generic case (five and two orbits, respectively). 


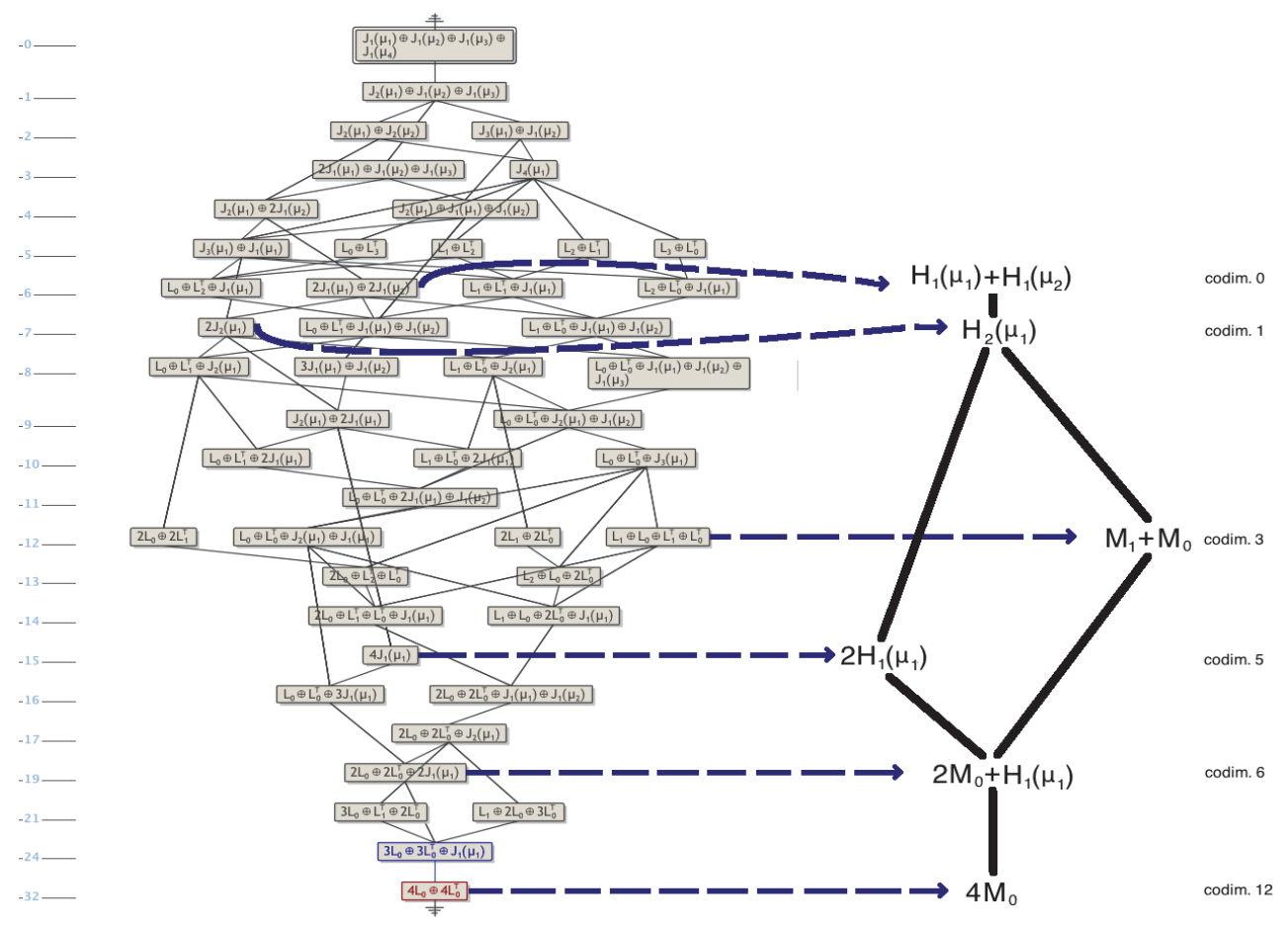

FIG. 3. Bundle stratification of skew-symmetric $4 \times 4$ matrix pencils under congruence (right graph) extracted from the bundle stratification of all $4 \times 4$ matrix pencils under strict equivalence (left graph).

Acknowledgments. The first author thanks Stefan Johansson and Pedher Johansson for introducing him to StratiGraph and for fruitful discussions. We are also grateful to the associate editor Froilán Dopico and the anonymous referees for the helpful suggestions.

\section{REFERENCES}

[1] D. L. Boley, The algebraic structure of pencils and block Toeplitz matrices, Linear Algebra Appl., 279 (1998), pp. 255-279.

[2] R. Byers, V. Mehrmann, And H. XU, A structured staircase algorithm for skewsymmetric/symmetric pencils, Electron. Trans. Numer. Anal., 26 (2007), pp. 1-33.

[3] T. J. Bridges And S. Reich, Multi-symplectic integrators: Numerical schemes for Hamiltonian PDEs that conserve symplecticity, Phys. Lett. A, 284 (2001), pp. 184-193.

[4] T. Brüll and V. Mehrmann, STCSSP: A FORTRAN 77 Routine to Compute a Structured Staircase Form for a (Skew-)Symmetric/(Skew-)Symmetric Matrix Pencil, Preprint 31, Institut für Mathematik, Technische Universität Berlin, 2007.

[5] J. Demmel and A. Edelman, The dimension of matrices (matrix pencils) with given Jordan (Kronecker) canonical forms, Linear Algebra Appl., 230 (1995), pp. 61-87.

[6] F. De Terán And F. M. Dopico, The solution of the equation $X A+A X^{T}=0$ and its application to the theory of orbits, Linear Algebra Appl., 434 (2011), pp. 44-67.

[7] F. De Terán And F. M. Dopico, The equation $X A+A X^{*}=0$ and the dimension of ${ }^{*}$ congruence orbits, Electron. J. Linear Algebra, 22 (2011), pp. 448-465.

[8] A. Dmytryshyn, Miniversal Deformations of Pairs of Skew-Symmetric Forms, arXiv:1104. $2492,2011$.

[9] A. Dmytryshyn, Miniversal Deformations of Pairs of Symmetric Forms, manuscript, arXiv:1104.2530, 2011. 
[10] A. R. Dmytryshyn, V. Futorny, and V. V. Sergeichuk, Miniversal deformations of matrices of bilinear forms, Linear Algebra Appl., 436 (2012), pp. 2670-2700.

[11] A. Dmytryshyn, V. Futorny, and V. V. Sergeichuk, Miniversal deformations of matrices under *congruence and reducing transformations, Linear Algebra Appl., 446 (2014), pp. 388-420.

[12] A. Dmytryshyn, V. Futorny, B. KÅgström, L. Klimenko, and V. V. Sergeichuk, Change of the congruence canonical form of 2-by-2 and 3-by-3 matrices under perturbations and bundles of matrices under congruence, Linear Algebra Appl., (2014).

[13] A. Dmytryshyn, S. Johansson, And B. KÅgström, Codimension Computations of Congruence Orbits of Matrices, Skew-Symmetric and Symmetric Matrix Pencils Using Matlab, Report UMINF 13.18, Department of Computing Science, Umeå University, Sweden, 2013.

[14] A. Dmytryshyn, B. KÅgström, and V. V. Sergeichuk, Skew-symmetric matrix pencils: Codimension counts and the solution of a pair of matrix equations, Linear Algebra Appl., 438 (2013), pp. 3375-3396.

[15] A. Dmytryshyn, B. Kågström, and V. V. Sergeichuk, Symmetric matrix pencils: Codimension counts and the solution of a pair of matrix equations, Electron. J. Linear Algebra, 27 (2014), pp. 1-18.

[16] A. Edelman, E. Elmroth, And B. K̊̊gström, A geometric approach to perturbation theory of matrices and matrix pencils. Part I: Versal deformations, SIAM J. Matrix Anal. Appl., 18 (1997), pp. 653-692.

[17] A. Edelman, E. Elmroth, And B. KÅgström, A geometric approach to perturbation theory of matrices and matrix pencils. Part II: A stratification-enhanced staircase algorithm, SIAM J. Matrix Anal. Appl., 20 (1999), pp. 667-699.

[18] E. Elmroth And B. K^̊gström, The set of 2-by-3 matrix pencils: Kronecker structures and their transitions under perturbations, SIAM J. Matrix Anal. Appl., 17 (1996), pp. 1-34.

[19] E. Elmroth, S. Johansson, And B. Kågström, Stratification of controllability and observability pairs: theory and use in applications, SIAM J. Matrix Anal. Appl., 31 (2009), pp. 203-226.

[20] F. R. Gantmacher, The Theory of Matrices, vol. 2, Chelsea, New York, 2000.

[21] P. Johansson, Software Tools for Matrix Canonical Computations and Web-Based Software Library Environments, Ph.D. thesis, Department of Computing Science, Umeå University, Sweden, 2006.

[22] S. Johansson, B. K̊̊gström, And P. VAn Dooren, Stratification of full rank polynomial matrices, Linear Algebra Appl., 439 (2013), pp. 1062-1090.

[23] P. Y. Li And R. Horowitz, Passive velocity field control of mechanical manipulators, IEEE Trans. Robotics Automation, 15 (1999), pp. 751-763.

[24] B. KÅgström, S. Johansson, And P. Johansson, StratiGraph Tool: Matrix Stratification in Control Applications, in Control and Optimization with Differential-Algebraic Constraints, L. Biegler, S. L. Campbell, and V. Mehrmann, eds., SIAM, Philadelphia, 2012, pp. 79-103.

[25] D. S. Mackey, N. Mackey, C. Mehl, and V. Mehrmann, Skew-symmetric matrix polynomials and their Smith forms, Linear Algebra Appl., 438 (2013), pp. 4625-4653.

[26] A. I. MaL'cev, Foundations of Linear Algebra, J. B. Roberts, ed., W. H. Freeman, San Francisco, 1963.

[27] P. J. Olver, Canonical forms for compatible biHamiltonian systems, in Solitons and Chaos, I. Antoniou and F. Lambert, eds., Springer-Verlag, New York, 1991, pp. 171-179.

[28] A. Pokrzywa, On perturbations and the equivalence orbits of a matrix pencil, Linear Algebra Appl., 82 (1986), pp. 99-121.

[29] L. Rodman, Comparison of congruences and strict equivalences for real, complex, and quaternionic matrix pencils with symmetries, Electron. J. Linear Algebra, 16 (2007), pp. 248-283.

[30] R. C. Thompson, Pencils of complex and real symmetric and skew matrices, Linear Algebra Appl., 147 (1991), pp. 323-371. 